\title{
REALIZACIÓN RETÓRICA DEL PROCESO DISCURSIVO Y DEL PRODUCTO TEXTUAL*
}

\author{
Lázaro Carrillo Guerrero**
}

\begin{abstract}
Resumen: El discurso, como proceso, y el texto, como producto, se desarrollan en una dimensión interaccional de naturaleza retórico-argumentativa. Donde la retórica del discurso tiene que ver con las prácticas sociales que desarrollan diferentes funciones comunicativas, y la retórica del texto con las voces que estructuran un significado para un auditorio. Estas retóricas están situadas en la ejecución de discursos multi-modales y multi-voces. De modo que, podemos definir al texto, en su selección de significados, como una unidad gramatico-retórica, y al discurso como una unidad de interacción entre realidad (y su construcción) y proceso cognitivo, estableciendo una dinámica argumentativa.

Palabras clave: discurso, texto, retórica, interacción comunicativa, registro, dinámica argumentativa.
\end{abstract}

\begin{abstract}
Discourse, as a process, and text, as a product, are developed in an interactional dimension of rhetoric-argumentative nature. Here the discourse's rhetoric has to do with the social practices that develop different communicative functions, and the text's rhetoric with the voices that structure a meaning for an audience. These rhetorics are situated in the performance of multi-modal and multi-voice discourses. Thus, we may define the text, from the meaning choice, as a grammatical-rhetoric unity, and the discourse as an interaction unity between reality (and her construction) and cognitive process, establishing an argumentative dynamic.
\end{abstract}

Key words: discourse, text, rhetoric, communicative interaction, register, argumentative dynamic.

\section{INTRODUCCIÓN}

Usar la lengua es establecer una relación de diálogo. Un diálogo entre la lengua y su contexto, entre la realidad y la realidad, y un diálogo donde el propósito fundamental del hablante es formular un mensaje para que funcione con efectividad como acto comunicativo. Y donde las características del auditorio, así como la percepción que el hablante tenga de éste y de la situación, intervienen en la construcción del texto y del discurso. Por tanto, usar la lengua es comunicar e interactuar con unas ideas, creencias, y emociones en unas situaciones determinadas. Y discurso es esa interacción, con una concreción textual. De modo que, el discurso, como proceso, y el texto, como producto, tienen en común esa

\footnotetext{
* Fecha de recepción: abril 2005

Fecha de aceptación y versión final: julio 2005

** Profesor, Instituto Galileo, Almería; $₫$ 1zro@msn.com.
} 
dimensión interaccional de naturaleza retórico-argumentativa. Donde la variabilidad y la adaptabilidad pueden considerarse como las grandes características comunicativas de nuestra conducta lingüística.

En todo este marco, la función esencial del sistema de la lengua, y por tanto de su gramática (Dik 1997), es pragmática, es decir: la función de ser un instrumento de interacción interpersonal. Y en todo este proceso de interacción comunicativa se contempla a la retórica como un "flujo de intercambios lingüísticos", a través de los cuales se comunica para obtener una efectividad en una acción retórica del discurso que incide, a la vez, sobre la representación de la realidad, y sobre las características internas del texto.

Por consiguiente, tanto el proceso discursivo como el producto textual se realizan desde una perspectiva retórica: lo probable, lo preferible, los mundos posibles; y con una determinada fuerza retórica, en las funciones conceptual, interpersonal, y textual de la lengua. Donde ambos, discurso y texto, poseen una estructura lingüística, y realizan unas funciones comunicativas en un contexto de interacción y en una situación comunicativa:

A text, I have sometimes suggested to enliven elementary lectures, is like a battlefield after the battle: the winning army is in possession, the losers have fled and the dead and wounded have been carried away. But the position and appearance of the winners and the traces of operations that can be seen on the battlefield may give the expert a chance of reconstructing something of the battle itself. So, too, the surface appearance of a piece of discourse may give clues to the conflicts and conspiracies that must have gone into its production. (Enkvist 1991: 13)

Esta particular descripción de Enkvist refleja metafóricamente que, ambos, texto y discurso son, respectivamente, producto y proceso $^{1}$ de una acción e interacción. Una interacción comunicativa, donde no se trata de vencedores y vencidos, sino de operaciones lingüísticas cargadas cognitiva, cultural e ideológicamente.

Halliday (1978: 60) manifiesta que el rasgo esencial del texto es la interacción, el proceso interactivo donde ocurren los intercambios de significados, siendo el texto el medio de intercambio. Él considera al texto (pág.125) como una forma linguiística de la interacción social; siendo el producto de muchas, simultáneas y sucesivas selecciones de significado, y realizado en una estructura léxico-gramatical.

Nystrand (1986: 39-48) no distingue entre texto y discurso, sino que a ambos los considera, primero una negociación de significados en el sentido de que se establece un marco mutuo de referencia, y segundo, un intercambio de significados cuando el entendimiento ha tenido lugar.

Es por tanto, el discurso un proceso comunicativo de interacción humana en íntima relación con el producto de esta interacción que es el texto. Y entendiendo que el texto funciona como un producto complejo del acto comunicativo y con fuerza propia. Ambas propiedades, complejidad y fuerza propia se deben a la característica esencial que hace al texto: la textualidad (propiedad mediante la cual, sucesivas oraciones forman un texto

\footnotetext{
${ }^{1}$ Los conceptos proceso y producto no son usados aquí en el sentido de la pareja Hjelmsleviana proceso/ producto, y como otros lingüistas lo han utilizado haciendo corresponder proceso a la experiencia del habla y producto a la experiencia de escribir. Aquí, texto es un producto escrito y hablado (sonoro)
} 
coherente). Y es en la textualidad donde queda plasmado este proceso de acción e interacción humana, es decir su discurso. Donde, Halliday entiende que la función del texto es realizar y significar ese proceso semiótico y multidimensional que es el discurso.

Desde la Semiótica Social, Hodge y Kress (1988:5-6) consideran al mensaje como la forma semiótica más pequeña, constituyente de dos planos: semiosic plane, mimetic plane. En el primero, el mensaje es orientado a un proceso semiótico, a un proceso social. En el segundo, el mensaje es acerca de algo, está conectado al mundo. Con todo esto, el mensaje tiene direccionalidad, tiene un origen y una meta, un contexto social y un propósito. De esta forma, ellos establecen que el texto tiene una orientación primaria al plano mimético, donde tiene significado en tanto que proyecta una versión de la realidad; y que el discurso se refiere más directamente al plano semiótico. Así, el texto y el discurso tienen una diferente orientación. Texto se refiere a la estructura de los mensajes socialmente adscritos, y siendo un objeto material concreto producido en el discurso. Discurso se refiere al proceso social en que los textos están incrustados. Hodge y Kress añaden que texto también se opone a otro importante concepto, sistema ${ }^{2}$. La semiótica ha desarrollado la noción de un sistema de signos como una estructura abstracta que es realizada o ejemplificada en el texto, y trata tales sistemas como estáticos, como hechos sociales. Un sistema está siendo constantemente reproducido y reconstituido en textos, de otra forma dejaría de existir. Así que los textos son, a la vez, la realización material de los sistemas de signos, y, también, el lugar donde el cambio tiene lugar continuamente. Y discurso, en este sentido, es el sitio donde las formas de organización social engranan con los sistemas de signos en la producción de textos, reproduciendo o cambiando así las clases de significados y valores que hacen una cultura. Ellos añaden que aunque discurso es enfáticamente una categoría social, esto no significa que texto y mensaje sean términos asociales. Texto y mensaje significan las relaciones sociales específicas en el momento de sus producciones o reproducciones.

Steiner y Veltman (1988: 1-3) consideran como sistemas, junto con la Pragmática, a los conceptos de Discurso y Texto, los cuales, a su vez, implican, respectivamente, unas dimensiones de descripción: proceso y producto. Al entender que la lengua tiene muchos niveles de organización, plantean que el término de "niveles" no es adecuado para estos conceptos y sobre todo cuando se trata de ingredientes vitales de descripción holística como son la Pragmática, el Discurso, y el Texto. Ellos prefieren considerarlos aspectos diferentes pero complementarios de las dimensiones de descripción. Estas dimensiones son respectivamente: Perspectiva, Proceso y Producto. Así, para ellos, estos sistemas: Pragmática, Discurso y Texto, asociados con estas dimensiones de descripción: Perspectiva, Proceso y Producto, juntos abarcan el punto de ataque de la lengua. Ellos definen estas realizaciones de la siguiente forma:

$\checkmark$ Pragmática es más una perspectiva que un genuino nivel o componente, como comúnmente se ha asumido, donde es contrastado, por lo general, con la semántica o gramática.

\footnotetext{
2 En contraste, este concepto, "sistema", ha sido, especialmente, utilizado por Firth, Halliday, y sus seguidores para referirse al conjunto de relaciones paradigmáticas en el uso de la lengua.
} 
Discurso es la lengua como proceso, y como tal está asociado con la clásica noción de "Retórica" son producidos y que responde de la organización interna de los textos.

$\checkmark$ Texto es el producto de la actividad de la lengua codificada en palabras y entregada al mundo en la sustancia de habla, escritura o signos.

Desde el punto de vista de la comunicación, Leech y Short (1981: 209-257) establecen la distinción entre la comunicación como discurso y la comunicación como texto. Discurso es la comunicación lingüística vista como una transacción entre hablante y oyente, como una actividad interpersonal cuya forma es determinada por su propósito social. Texto es la comunicación lingüística (hablada o escrita) vista simplemente como un mensaje codificado en su medio auditivo y visual, pero se sitúa dentro de esa transacción comunicativa, entre hablante y oyente, que es el discurso. Ellos hablan de la retórica del texto y de la retórica del discurso; usando el término retórica en el sentido tradicional del "arte o destreza de comunicación efectiva". La retórica del texto trata con la forma expresiva y superficial de la lengua, determinada por la sintaxis, fonología y grafología, y estando dentro del estilo sintáctico, fonológico/grafológico. La retórica del discurso es la manera en que el mensaje es usado para lograr sus propósitos tales como informar, persuadir, dar órdenes, etc.

Con todo, entendemos que la retórica del discurso tiene que ver con las prácticas sociales que desarrollan diferentes funciones comunicativas, y que la retórica del texto con las voces que estructuran un significado para un auditorio. Así, en el texto religioso de oración colectiva (The Cathedral Church of the Blessed Virgin Mary in Salisbury) que se puede encontrar, repartido por los bancos, en la catedral de Salisbury, la retórica del discurso viene dada por toda la participación colectiva, ritualizada, a través del texto y su significado extraído individual y colectivamente; y la retórica del texto viene dada por toda su estructuración textual de acuerdo con el mensaje o significado que se pretender establecer (religioso, de creencias,...) según una determinada intención comunicativa.

Estimamos que estas retóricas están situadas en la perspectiva que, desde el análisis del discurso, plantean Jaworski y Coupland (1999: 7-8). Ellos hablan de discursos multimodales y multi-voces. Refiriéndose con ello a que siendo el discurso un conjunto de prácticas sociales que construyen el significado, muchos de los textos producidos en este proceso son multi-modales, es decir, hacen uso de más de un sistema semiótico. Y los textos a menudo reflejan y reciclan diferentes voces o heteroglossia (Bakhtin 1986), las cuales pueden realizarse a través de diferentes modalidades o de una sola modalidad, y dirigiéndose a uno o a muchos auditorios.

\footnotetext{
3 "The source of interest in discourse and text studies has classically been in the field of RHETORIC, both as an academia discipline and as a profession. Current rhetoric is rediscovering linguistics as a provider of more explicit tools and explanations than are otherwise available... We are witnessing the re-emergence of a union between rhetoric on the one hand, and grammar and philosophy on the other, in the explicit recognition of language as the prime medium of the processes which are studied by these disciplines..." (Steiner and Veltman 1988: 3).

"La retórica es una capacidad lingüística relacionada con aspectos comunicativos y cognoscitivos que emplea principios que no son ni estructurales ni sintagmáticos” (Martínez-Dueñas 2002: 26).
} 
Kress et al. (1997: 257-9) hablan, también, del fenómeno de Multi-modality, como un fenómeno que no es nuevo, pero que se ha desarrollado últimamente más. Se trata de la conjunción en el texto de otros modos (visual, gestual, sonoro, etc.) de representación y comunicación, además del propio texto ${ }^{4}$. Según ellos, esto entraña un movimiento en la investigación sistemática: desde la linguiística a la semiótica, desde la lengua a todos los modos de representación invocados en el texto. Y desde esta perspectiva, afirman que la ideología es un factor que está involucrado en todos los modos representados en el texto ${ }^{5}$.

\section{TEXTO}

El texto es esencialmente una unidad semántica, que tiene que ser considerada desde dos perspectivas al mismo tiempo, como producto y como proceso (Halliday y Hasan 1985). Cualquier extensión de lengua puede ser considerada como un texto. Y éste puede ser hablado o escrito, producto de un monólogo o de la interacción de varias personas. Sin que esté definido ni por su tamaño, ni por su forma ${ }^{6}$. De modo que, con el término de texto podemos hacer referencia tanto a un acto de habla, a un intercambio oral entre participantes, a un pasaje o episodio, como a un macro-acto de habla, una narrativa completa, etc.

Brown y Yule (1983:4-11) tratan la noción de texto como la representación del discurso, donde el registro verbal de un acto comunicativo puede ser tanto escrito como hablado, y aclarando que:

- un texto escrito puede ser presentado en diferentes ediciones, tipografías, tamaños de papel, en una o dos columnas, y ser todas distintas representaciones del mismo texto,

- y que la grabación de un texto hablado puede recoger además del texto otros elementos sonoros que no constituyen parte del texto sino del contexto.

Además, no podemos considerar que un texto está compuesto de oraciones, sino que está construido, realizado por oraciones (Halliday 1978: 133-5; Quirk et al. 1985: 1423; Fowler 1996: 80). Y donde estas oraciones que realizan el texto son hasta cierto punto mutuamente predictivas unas de otras (Lemke 1991: 23). O dicho de otra manera, tal como Sinclair (1994) lo expresa:

\footnotetext{
${ }^{4}$ Un ejemplo de ello lo representa el texto de la página web (en Internet): Low Tar Exponed: the truth about smoking 'lights' and 'mild', [http://www.lowtarexposed.org/], donde el texto se entremezcla con imágenes (fijas, y en movimiento) y sonidos. Todo a disposición de los usuarios, quienes mediante sus selecciones (de acuerdo con sus intereses, actitudes, etc.) construirán, en él, su propio texto.

${ }^{5}$ Kress y Leeuwen (2001) definen a este texto "multimodal" como un texto que utiliza varios modos de comunicación de forma integrada (por ejemplo, texto hablado, texto escrito, imágenes) para transmitir un mensaje. Matizando que esta multimodalidad es una caracteristica actual de muchos textos.

6 "The word TEXT is used in linguistics to refer to any passage, spoken or written, of whatever length, that does form a unified whole... A text may be spoken or written, prose or verse, dialogue or monologue. It may be anything from a single proverb to a whole play, from a momentary cry for help to an all-day discussion on a committee. A text is a unit of language in use. It is not a grammatical unit, like a clause or a sentence; and it is not defined by its size" (Halliday and Hasan 1976: 1).

- "By way of ostensive definition, 'text' means Faulkner's speech of acceptance of the Nobel prize; or Hemingway's The Old Man and the Sea; or the twenty-five-minute discussion of the members of the press with Undersecretary of Stage George W. Ball, on April 10, 1966, 11:30-12:00 (CST); or Shakespeare's Othello; or the single sentence 'Fire' screamed by a hotel occupant running from a building; or Toynbee's twelve volumes of A Study of History" (Kinneavy 1971: 22).
} 
...each sentence contains one connection with other states of the text preceding it. That is to say it contains a single act of reference which encapsulates the whole of the previous text and simultaneously removes its interactive potential. The occurrence of the next sentence pensions off the previous one replaces it and becomes the text. The whole text is present in each sentence. The meaning of each previous sentence is represented simply as part of the shared knowledge that one is bringing to bear in the interpretation of a text at any point (Sinclair 1994: 17).

Por tanto, considerando esta función constructiva o explorativa que la oración tiene en el texto, que además de hacia atrás, también puede ser hacia adelante, y considerando que cada sucesiva oración es, por un momento, el texto entero, se le puede asignar a la oración dos funciones básicas con respecto al texto:

- una la función constructiva del texto

- y otra la función comunicativa dentro del texto.

Siendo estas funciones, constructiva y comunicativa de las oraciones, las que conforman la cohesión textual de un texto; en una asociación de, y proceso comunicativo de reglas gramaticales y principios pragmáticos ${ }^{7}$.

Halliday y Hasan (1976) asocian el concepto de cohesión ${ }^{8}$ al concepto de textura, la cual deriva del hecho de que el texto funciona como una unidad con respecto a su entorno. Pero además, funciona, no como una unidad gramatical ${ }^{9}$, sino como una unidad semántica (Halliday 1994: xvii) con respecto a los recursos por encima de la oración, y con respecto a las unidades por debajo de ella.

Para Halliday y Hasan (1976) es importante subrayar que la cohesión es una relación semántica que se refiere a las relaciones de significado que existen dentro del texto y que lo definen como un texto; y es realizada a través del sistema lexico-gramatical: parcialmente a través de la gramática (cohesión gramatical) y parcialmente a través del vocabulario (cohesión léxica).

Adam (1997: 20 y sigs.) considera al texto como una configuración reglada por diversos módulos o subsistemas en una interacción constante:

- tres constituyendo la configuración pragmática: 1) la acción ilocutiva, 2) las referencias enunciativas, 3) y la representación construida o mundo del texto (organización semántico-referencial),

- y dos constituyendo la conexión textual u organización general: 4) la gramática de la oración, 5) y la gramática del texto.

\footnotetext{
${ }^{7}$ Para un estudio práctico de ello, ver a Martínez-Cabeza (2002). Y para un estudio metódico de este proceso lingüístico-comunicativo desarrollándose desde la palabra hacia la oración y hacia el texto, ver a Halliday (2002a: parte 2).

${ }^{8}$ Halliday y Hasan (1976: 294-5) sugieren que es razonable hacer uso del concepto de cohesión como criterio para el reconocimiento de los límites de un texto, así podríamos decir si cierto pasaje constituye un texto o tenemos que tratarlo como dos o más textos. Pero, pueden haber muchos casos indeterminados, afirman, y la adecuada y explícita definición de texto puede no proporcionarnos un criterio automático para reconocer en todos los casos lo que es un texto y lo que no lo es.

Halliday (1978: 141) afirma que texto es un concepto indeterminado: puede ser muy largo o muy corto; y puede tener los límites no muy claros.

9 "But meanings are realized through wordings; and without a theory of wordings — that is, a grammar there is no way of making explicit one's interpretation of the meaning of a text" (Halliday 1994: xvii).
} 
Él define al texto mediante una estructura secuencial que le permite abordar la heterogeneidad compositiva en términos de jerarquía: paquetes de proposiciones (macro-proposiciones), constituidas de $n$ proposiciones (pág.29). De esta forma, la unidad textual que él designa mediante la noción de secuencia puede ser definida como una estructura:

\section{[ \# T \# [Secuencia(s) [macro-proposiciones [proposicion(es)]]]]}

Adam $^{10}$ caracteriza los tipos de texto a través de estas configuraciones lingüísticas, fijadas ya de antemano. Es una caracterización, más bien, desde el interior, desde el mismo texto, y no desde el contexto. Ya que se caracteriza una organización de unidades lingüísticas independientes de las condiciones en que su respectivo discurso ha tendido lugar; es decir, independientes de su registro.

Beaugrande y Dressler (1981: 3) definen el texto como "a communicative ocurrence". Y establecen que un texto a diferencia de un texto no comunicativo y por consiguiente un no texto, debe de poseer, sin faltar ninguno, siete grados de textualidad: Cohesión, Coherencia, Intencionalidad, Aceptabilidad, Informatividad, Situacionalidad e Intertextualidad. He aquí un extracto de la definición de todos estos conceptos:

"Cohesion,... the ways in which the components of the SURFACE TEXT ${ }^{11}$, i.e. the actual words we hear or see, are mutually connected within a sequence.

Coherence,,.. the ways in which the components of the TEXTUAL WORLD, i.e. the configuration of CONCEPTS and RELATIONS which underlie the surface text, are mutually accessible and relevant.

Cohesion and coherence are text-centred notions, designating operations directed at the text materials. In addition, we shall require user-centred notions which are brought to bear on the activity of textual communication at large, both by producers and by receiver.

Intentionality, concerning the text producer's attitude that the set of occurrences should constitute a cohesive and coherent text instrumental in fulfilling the producer's intentions, e.g. to distribute knowledge or to attain a GOAL specified in a PLAN.

Acceptability, concerning the text receiver's attitude that the set having some use or relevance for the receiver, e.g. to acquire knowledge or provide co-operation in a plan.

Informativity,... the extent to which the occurrences of the presented text are expected vs. unexpected or known vs. unknown/certain.

Situationality,... the factors which make a text RELEVANT to a SITUATION of occurrence.

Intertextuality,... the factors which make the utilization of one text dependent upon knowledge of one or more previously encountered texts" (Beaugrande y Dressler 1981: 3-11).

Frente a esta dialéctica de texto / no texto, Edmonson (1981: 12-14) prefiere hablar de textos antigramaticales o no logrados, antes que de no textos. Edmonton, además, comen-

\footnotetext{
${ }^{10}$ Adam (1997) retoma y desarrolla en Francia la tipología de E. Werlich (1982), pero considerando a la tipología secuencial como un punto de vista parcial sobre un objeto heterogéneo (el texto).

11 "The surface text is the set of expressions actually used; these expressions make some knowledge explicit, while other knowledge remains implicit, though still applied during processing" (Beaugrande 1984: 36).
} 
ta que todos nosotros siempre estamos utilizando procedimientos interpretativos y que el material suprimido de un texto puede ser recuperado. A esto hay que añadir la noción de coherencia como proceso mental, reflejada en Gernsbacher y Givón (1995) donde queda de manifiesto que coherencia es el resultado de las operaciones mentales sobre el texto. Se trata de un fenómeno cognitivo de la mente que produce y comprende el texto (Givón 1995b: 59-115). Y así, nos podemos encontrar con un texto lleno de inconsistencias y con muy escasos marcadores de cohesión y ser, mediante este proceso cognitivo, un texto coherente. También puede darse el caso contrario, un texto con ninguna inconsistencia y suficientes marcadores de cohesión, y ser un texto incoherente. Y desde esta perspectiva, podemos concluir con Cook (1989: 75) en afirmar que la coherencia se crea mediante nuestra interacción con el texto, y, conjuntamente, mediante el emisor y el receptor del texto. Ante esta cuestión, Fowler (1996: 80-81) aclara que a través de la cohesión, un texto establece y desarrolla racionalmente un tema e indica cuales son las partes prominentes y las subordinadas de un argumento o una historia, y que estando estructurado de esta manera, emergerá para el lector como coherente.

En definitiva, el contenido, la extensión y las partes de un texto (o discurso) vienen definidas por su acción o interacción comunicativa. Estando, así, el texto marcado por su acto comunicativo: donde empieza y termina el acto comunicativo allí empieza y termina el texto, independientemente de los participantes. Un ejemplo muy ilustrativo de ello lo muestra el texto de la página $w e b^{12}$, Low Tar Exponed: the truth about smoking 'lights' and 'mild', situada en el contexto interactivo de Internet. Donde la página principal y todas sus páginas asociadas a ella de acuerdo con el tema, "la nocividad del tabaco", conforman un texto, una unidad semántica. Pero, donde cada usuario construirá, de acuerdo con sus selecciones (pinchando con el ratón) y con sus intereses, su propia unidad semántica, su propio texto, que podrá coincidir o no con la unidad semántica de la página $w e b$, y con la que construyan otros usuarios.

Halliday (1978: 133-5) establece tres factores en la construcción de un texto: estructura genérica, estructura textual (temática e informativa), y cohesión.

La estructura genérica es la forma que un texto tiene como una propiedad de su género; está fuera del sistema lingüístico, en la estructura semiótica, en un nivel más alto. Todo tipo de texto o discurso, incluida la conversación más espontánea e informal, tiene estructura genérica, la cual puede ser llevada dentro del marco del concepto de registro.

La estructura textual engloba los recursos específicos del sistema de la lengua en la formación del texto; sus opciones temáticas, realizadas a través de la oración, contribuyen a la estructura lexico-gramatical, y sus opciones informativas, realizadas en el sistema fonológico a través del grupo tónico, contribuyen a la estructura de la información. Aquí, Halliday matiza al componente textual como un componente de significado, junto con los componentes conceptual (ideational) e interpersonal, y al texto como una unidad semántica definida por el componente textual. Entendiendo que, por encima del texto, hay diferentes niveles semánticos más altos, y estableciendo así que un texto, además de ser realizado en los niveles más bajos del sistema lingüístico, lexico-gramatical y fonológico, es también realizado en estructuras semióticas de nivel más alto con sus propios modos de interpretación, literario, sociológico, psicoanalítico, etc.

12 http://www.lowtarexposed.org/ 
El componente textual que Halliday (1978) utiliza para definir al texto como una unidad de significado, es el mismo concepto de cohesión que Halliday y Hasan (1976) definen como una relación de significado y no estructural; y al cual utilizan también para definir al texto como una unidad semántica, y no una unidad estructural. Halliday y Hasan afirman (págs.293-4) que esta unidad semántica es una unidad de significado en un contexto, relacionada como un todo al ambiente en el cual está emplazada. Presentando así, esta relación, una forma de consistencia que es definida mediante el concepto de registro. Y es, según ellos, esa consistencia o coherencia, en los estilos de significado o tipos de configuración semántica, la que sostiene su relación a su entorno o medio ambiente.

Por tanto, el texto, a diferencia de la oración, no es una unidad gramatical sino una unidad semántica y pragmática: cohesionando en su contexto del mundo real, semántica y pragmáticamente, y siendo también coherente interna o lingüísticamente (Quirk et al. 1985: 1423). De modo que esta perspectiva pragmática del texto es una cuestión retórica:

- El texto es una forma lingüística de interacción social, un producto de su entorno, funcionando en ese entorno, y un continuo proceso, un proceso socio-semántico, donde hay una constante relación de desplazamiento entre el texto y su entorno, paradigmática y sintagmáticamente (Halliday 1978: 125/139).

- El texto es una unidad de organización semántico-situacional: un continuum de significado en contexto, construido alrededor de la relación semántica de cohesión y de acuerdo a la particular configuración situacional-semántica o registro (Halliday y Hasan 1976: 25).

En consecuencia, se puede definir el concepto de texto como una unidad semánticopragmática: una unidad de significado y una unidad de lengua en uso; realizada en un acto comunicativo, y codificada en una estructura gramatical y situacional. Cuyo significado es potencial (los textos no tienen un significado determinado intrínseco), en contraste con los significados interpretativos que los destinatarios realicen en los determinados actos comunicativos. En definitiva, se trata de una unidad gramatico-retórica (se construye una unidad semántica de acuerdo con unos objetivos comunicativos), y no de una unidad gramatical como una oración, ni de una unidad de forma.

En palabras de Halliday (1978: 109) ${ }^{13}$, el texto representa selecciones y puede ser definido como el significado potencial actualizado. De manera que el texto funciona como un sistema actual, y lengua como un sistema virtual (Beaugrande 1980; Beaugrande y Dressler 1981: 35).

Fairclough (1995: 18) considera el texto como una selección de opciones desde un potencial disponible, y establece dos niveles de selección: a) uno entre lo disponible del lenguaje, desde el potencial gramatical y léxico, lo cual está unido al otro, b) a qué géneros recurrir al producir (o interpretar) un texto y que discursos usar. Identifica tres aspectos del texto que siempre están presentes de alguna forma: social identities, social relations, and systems of knowledge and belief (Fairclough 1955: 55).

13 Halliday (1978: 122) reajusta los conceptos de competence/performance en su noción meaning potential refiriéndose al nivel del código de la lengua y al nivel de la semántica. Él afirma que el texto es una continua progresión de significados. Considerando que los significados son las selecciones hechas por el hablante desde las opciones que constituye el potencial del significado, y que el texto es la actualización de este potencial de significado. 
En todo este proceso de actualización, la comunidad comunicativa hace que este proceso sea muy similar entre todos sus miembros (Beaugrande y Dressler 1981:36), y a su vez, este proceso estaría regulado por lo que Beaugrande (1980) llama el sistema cibernético constituido por el texto:

.... text constitutes a CYBERNETIC system which continually regulates the functions of its constituent occurrences. Whenever a textual occurrence falls outside the participants' systems of knowledge about language, content, and purpose, the STABILITY of the textual system is disturbed and must be restored by REGULATIVE INTEGRATION of that occurrence, e.g. via additions or modifications to one's store of knowledge. (Beaugrande 1980; en Beaugrande y Dressler 1981: 36)

Y podemos considerar a esta integración regulativa como una eficaz acción retórica, donde los principios regulativos, que Beaugrande y Dressler definen, controlan la comunicación textual:

We envision at least three regulative principles. The EFFICIENCY of a text depends on its use in communicating with a minimum expenditure of effort by the participants. The EFFECTIVENESS of a text depends on its leaving a strong impression and creating favourable conditions for attaining a goal. The APPROPRIATENESS of a text is the agreement between its setting and the ways in which the standards of textuality are upheld. (Beaugrande $y$ Dressler 1981: 11)

Control de comunicación textual y acción retórica que se realizan en un proceso cognitivo, a través de las intenciones del hablante y de la interpretación de estas intenciones por el oyente (o auditorio). Y donde podemos hablar, de acuerdo con Blakemore (2002), de la realización de una relevancia comunicativa que marca discursivamente: una lógica a través de comparaciones y contrastes (similarly, in addition, by contrast, let me give you an example, such as..., to illustrate this point... etc.), la inferencia por parte del auditorio (but, however, etc.), unos argumentos (I point this out because..., due to..., this leads/brings me to... etc.), una secuencia (first, secondly, finally, etc.), una focalización (so far we have looked at..., now..., let's now move to..., etc.), etc.

Dentro de esta misma perspectiva (comunicación textual y acción retórica como proceso cognitivo), Widdowson (2004: capítulo 5), explorando las relaciones entre gramática, texto y discurso, introduce la noción de "pretexto" como un factor adicional en el proceso interpretativo. Y Sanders y Gernsbacher (2004) muestran como el fenómeno de la "accesibilidad" al significado (textual y discursivo) constituye un proceso lingüístico y psicolingüístico. Donde, por ejemplo, las formas lingüísticas más largas tienden a usarse cuando los referentes son relativamente de baja accesibilidad, y las formas lingüísticas más cortas tienden a usarse cuando los referentes son altamente accesibles.

Ante este panorama, podemos concluir que la actualización de la lengua es realizada en el proceso discursivo y mediante el texto. Y que éste, el texto, tanto en la producción como en la recepción implica una selección de significados en dos niveles o sistemas: el gramatical, determinado por el código de la lengua; y el retórico, determinado por la situación discursiva. Lo ilustramos de la siguiente manera: 


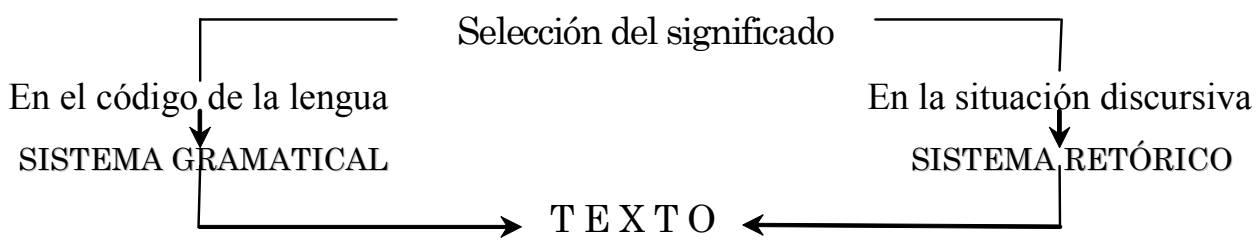

Figura 1. La actualización del texto mediante la selección de significados

Así, en el texto de un mensaje dejado en el servicio del contestador telefónico, la hablante Thèrese, de acuerdo con la situación discursiva que se le presenta al no estar su interlocutor al otro lado del teléfono, hace unas selecciones, según su relación de amistad con su interlocutor, en el sistema retórico para dejar su mensaje: saluda y se da conocer [Hello Lázaro, it's Thèrese], anuncia que llamará más tarde [I'll try you again later], descarta que el motivo de su acto comunicativo sea algo preocupante, sino que es más bien algo rutinario [Nothing important], se despide con amabilidad [Thank you!, bye!]; todo ello con una disposición declarativa, y exclamativa al final, de cortesía. A su vez, estas selecciones intervienen también en el sistema gramatical: construyendo un texto con oraciones simples independientes entre sí, en una relación paratáctica, sin ningún conector entre ellas; con un dinamismo comunicativo progresando desde los elementos ya conocidos [...it's Thèrese] hacia la información de valor más alto, el tema de la llamada [Nothing important]; y la selección, en la segunda oración [I'll try you again later], de unos elementos deícticos relevantes usados anafóricamente: I, you, later.

\section{DISCURSO}

El término "discurso" puede ser utilizado para referirse a una determinada producción textual asociada a un escenario particular. Así, se puede hablar de "discurso religioso", "discurso político", "discurso didáctico", "discurso publicitario", etc. De esta forma, discurso es definido por el contexto de producción de los textos y por su carácter de género (Mills 1997: 159). Por otro lado, Werth (1999) propone la siguiente definición: “...the discourse is an actually occurring piece of language whose dimensions are dictated by the situation (including, crucially, the participants in it). A discourse is therefore essentially a 'megautterance', and utterances are perceived sub-units of it"(Werth 1999: 3).

Por tanto, el discurso, al igual que el texto, no es un simple conjunto de expresiones, sino que posee una unidad caracterizada principalmente por tres aspectos: es construido con un propósito, conforma una organización y una coherencia, y pertenece a una cultura y a una situación social.

Schiffrin (1994: 20-43) examina tres definiciones de discurso: una derivada del paradigma formalista, otra del paradigma funcionalista, y una tercera, propuesta por ella, que es una confluencia entre las dos primeras. Para la definición formalista ${ }^{14} \mathrm{o}$ estructuralista, ella, elige la dada por Stubbs (1983:1) quien afirma que discurso es: "language above the

\footnotetext{
${ }^{14}$ Los formalistas ven a la lengua como un sistema autónomo, donde la lengua puede tener funciones sociales y cognitivas pero que estas funciones no afectan a la organización interna del lenguaje.
} 
sentence or above the clause". En la definición funcionalista ${ }^{15}$, el discurso es considerado como un sistema, un uso de la lengua o forma de hablar social y culturalmente organizado. Y su definición de discurso la asienta en una interacción entre estructura y función: "discourse is utterances". Con ello, Schiffrin conecta la idea de que discurso es lengua por encima de la oración con la noción de contexto, ya que asume que utterance a diferencia de la oración, es una unidad de uso de la lengua contextualizada.

Siguiendo la teoría estructuralista (Ronald Barthes, Tzvetan Todorov, Gérard Genette), Chatman (1978: 19-31), al analizar los componentes de la narrativa, sitúa al discurso como una de las dos partes de la narrativa (la otra es el contenido o historia); y lo define como la expresión formal, el medio mediante el cual el contenido es comunicado, el "cómo" es comunicado. A este tratamiento de Chatman y los estructuralistas, se le puede relacionar la idea, basada en la "Teoría de los actos de habla" y desarrollada por Austin y Searle, de que una oración se constituye en dos componentes: fuerza y contenido. Y es esta fuerza la que conforma el nivel del discurso. Así, en palabras de Sinclair (1992: 79), la expresión "It's getting late", dependiendo de cómo es expresada y en qué contexto, puede adquirir el valor de una amenaza, una advertencia, una insinuación, una queja, etc., quedando su significado convencional sin ser afectado. Es obvio añadir que este nivel del discurso también tiene que ver, además de con el contexto, con los niveles de forma y contenido. Y ello nos lleva a la conclusión de que tanto la historia que es comunicada, su contenido, como el "cómo" es comunicado, su fuerza, constituyen los pilares (uno semántico y el otro pragmático) sobre los que todo discurso se sustenta.

Está claro que el discurso tiene que ver con el funcionamiento comunicativo, y siendo así, toda unidad comunicativa tendrá su propio discurso. Dentro del campo de la Etnografía de la Comunicación ${ }^{16}$, Hymes (1986: 56-9) sugiere tres unidades de interacción comunicativa, en mutua relación jerárquica: speech acts que forman parte de speech events, y éstas, a su vez, formando parte de speech situations ${ }^{17}$.

Y desde esta función comunicativa, podemos considerar que esta relación jerárquica marca las unidades en la que el discurso se realiza. A las cuales se podrían añadir las nociones de Edmonson (1981: 201-204) de: discourse world ${ }^{18}$, world shift, coexisting discourse-worlds.

\footnotetext{
${ }^{15}$ Los funcionalistas ven a la lengua relacionada con su función social: la lengua tiene funciones que son externas al mismo sistema lingüístico y estas funciones externas influencian la organización interna del sistema lingüístico.

${ }^{16}$ La etnografía de la comunicación estudia la sociolingüística de la lengua, considerando que su uso está relacionado a los valores sociales y culturales. Se reconoce a Dell Hymes el padre de este enfoque. Él inició y desarrolló, en la década de 1960, numerosos estudios de modelos de comunicación en varias sociedades alrededor del mundo.

17 Por ejemplo, una broma-chiste puede ser un speech act que a su vez es parte de una conversación (speech event) la cual tiene lugar en una fiesta (speech situation). Es también posible que un speech act sea el completo y único speech event en una speech situation. En el primer ejemplo podemos ver un discurso dentro de otro discurso, y ambos dentro de la misma situación; pero, a ambos los diferencian su fuerza y el cómo son comunicados.

18 La noción de discourse world puede identificarse con la de speech community, en cuanto que se refiere a la particular manera de hablar de un grupo de gente que se identifica usando palabras, expresiones y maneras de interactuar que los sitúan aparte. También están relacionados con jargon register.

De acuerdo con Werth (1999: 51), se trata de ese conocimiento conceptual que los participantes necesitan ser capaces de representar para afrontar un discurso.
} 
A discourse world is to be understood as an application of the notion of a possible world derived from logical semantics to the pragmatic interpretation of conversational behaviour. A discourse world is then a function from interactional act to communicative act: the locutionary act requires an illocutionary value by reference to an operant discourse world. (Edmonson 1981: 201)

Edmonson define world-shift como la noción que nos permite interpretar una expresión funcionando en más de un acto comunicativo. Este acto comunicativo puede ser caracterizado de forma diferente con respecto a los oyentes que reciben la misma expresión. Y co-existing discourse worlds es un conjunto de funciones (discourse worlds), desde el acto interaccional hasta el acto comunicativo, donde se opera, y a menudo, simultáneamente.

En esta complejidad de interacciones, Fawcett (1980: 255-6) sugiere que el discurso es esencialmente una estructura semiótica, más bien que lingüística. Para él, una unidad de discurso puede ser definida en términos del tema que trata y del conjunto de intenciones socio-sicológicas ("funciones") asociadas con ella. Un acto del discurso no está necesariamente codificado en lengua. Las intenciones socio-psicológicas que un ejecutante asigna a su acto semiótico puede influenciar las selecciones en la red de la fuerza ilocutiva, sin ser idénticas con aquellas selecciones.

Fairclough (1995: 2-18) usa el término discurso para referirse al lenguaje escrito y hablado, y además a otros tipos de actividad semiótica — actividad que produce signos, tales como imágenes visuales (fotografía, cine, video, diagramas,...) y comunicación no verbal - gestos... (aquí añadiríamos a los datos de Fairclough, conductas rituales en actos y eventos comunicativos; así, el texto religioso de oración colectiva (The Cathedral Church of the Blessed Virgin Mary in Salisbury) que se puede encontrar, repartido por los bancos, en la catedral de Salisbury, nos muestra estas conductas rituales, tales como cánticos, ponerse de pie o arrodillarse, declamar y responder al unísono, etc.). En esta línea, Fairclough considera el discurso como una forma de práctica social ${ }^{19}$, y en él distingue dos principales sentidos. Uno tiene que ver con los estudios sobre la lengua: el discurso como acción social e interacción, la gente interaccionando en situaciones sociales reales. El otro tiene que ver con la teoría social post-estructuralista: el discurso como una construcción social de la realidad, una forma de conocimiento. Su concepto del discurso asume estos dos sentidos juntos, estando el primer sentido más íntimamente asociado con la función interpersonal de la lengua y con el concepto de género, y el segundo más íntimamente asociado con la función conceptual de la lengua y con los discursos.

A estos dos sentidos del discurso de Fairclough, los cuales serían considerados como partes del proceso del discurso, habría que añadir el producto del discurso: el texto íntimamente asociado con la función textual. De modo que podríamos trazar el siguiente esquema:

\footnotetext{
19 En un estudio reciente, Fairclough (2003) propone el análisis textual y el análisis del discurso como medio de investigación social.
} 


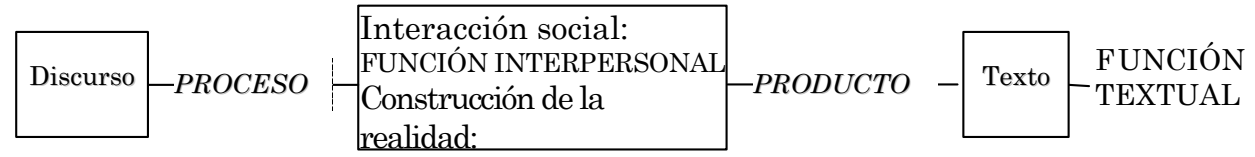

Figura 2. Relación discurso-texto en las funciones de la lengua

Hay que aclarar que el texto como producto del discurso refleja también las funciones conceptual e interpersonal. Y, de acuerdo con Halliday (1978), las funciones conceptual, interpersonal y textual de la lengua están siempre operando en cualquier texto, e incluso en cualquier frase u oración.

La relación establecida en esta Figura 2. Relación discurso-texto en las funciones de la lengua, puede ir también en sentido inverso, es decir desde el texto hacia el discurso; por ejemplo, cuando leemos u oímos un texto, lo entendemos porque hemos ido hacia la comprensión de su discurso. En este sentido, Carter (1997: 118) define el discurso como la realización pragmática de la acción social, conduciendo ello a reconocer que de un mismo texto pueden derivarse diferentes discursos. Desde esta perspectiva, se hace evidente la existencia de una pluralidad en textos y discursos. Y en esta misma línea, Carter (1997: 120) recoge la definición de discurso de Birch: "Discourse is about interaction and exchange; about people and institutions; about power, status and control" (Birch 1996: $65)$.

Sin embargo, Edwards (1997) deja a un lado la noción del discurso como comunicación, y, desde la perspectiva del cognitivismo, considera las categorías y funciones de la mente como categorías del discurso, las cuales son acuñadas y desplegadas en la acción que el discurso y el texto realiza. Esta acción es considerada como una actividad social.

Edwards caracteriza el discurso de la siguiente forma: "It is the business of discourse to formulate and deal with the nature of the world outside and the world within: with reality and mind, and the relations between them" (Edwards 1997: 19). Y enfoca el discurso desde tres direcciones: lengua, representación mental y comunicación (Edwards 1997: 24).

De esta forma, Edwards considera que el discurso es un asunto de cognición y realidad. Pero, la realidad y su construcción por un lado, y el proceso cognitivo y la realidad por otro, requieren interacción. Una interacción comunicativa manejada por el discurso. Lo cual representamos así:

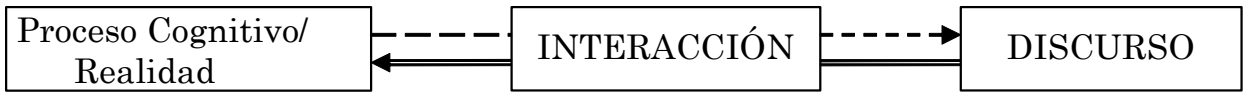

Figura 3. Interacción realidad, cognición, y discurso

La interacción entre el proceso cognitivo y realidad nos conduce a una interacción en el discurso; pero, a su vez, hay un flujo en la dirección contraria, el discurso maneja la interacción comunicativa y la interacción proceso cognitivo / realidad. 
El discurso que genera el texto religioso de oración colectiva (The Cathedral Church of the Blessed Virgin Mary in Salisbury) repartido por los bancos en la catedral de Salisbury, muestra claramente este proceso: primero se requiere un proceso cognitivo y una interacción en los participantes para establecer y desarrollar el evento comunicativo religioso, pero a su vez, el discurso establecido mediante el texto maneja la interacción comunicativa de todos los participantes, e interviene en sus procesos cognitivos de construcción de una "realidad" religiosa, de fe o de creencias. En Virtanen (2004) este proceso cognitivo ocupa el interés central en la interacción comunicativa que se establece a través del texto y del discurso.

Givón al hablar de discurso habla también de proceso mental y proposición. Manifiesta (1984: 239) que la comunicación humana es multi-proposicional y que otra forma de referirse a ello es usar el nombre de discurso. Así, discurso es un "híbrido" o compromiso informativo donde cada proposición, en la cadena del discurso, añade alguna información, y donde la dicotomía nueva/vieja o información foreground/background es una consecuencia del requerimiento de coherencia de un discurso multi-proposicional. Givón (1984: 242) añade que esta característica acumulativa del discurso - añadiendo nueva información a la pre-existente- se aplica tanto al discurso específico como un sistema coherente, como al conocimiento genérico, también, como un sistema coherente. Él reinterpreta la gramática (1984: 893) como un proceso mental de instrucciones, la cual no interactúa directamente con el texto en el comportamiento comunicativo, sino que manipula (o es desplegada por) la mente, que en cambio, interpreta o produce el texto. Y afirma que el discurso es producido en una interacción entre dos perspectivas, la del hablante y la del oyente; y aquí, la interacción ocurre en la mente de ambos participantes. Cada uno, además de su propia perspectiva, también intenta construir algún modelo de la perspectiva del otro.

Halliday (2002b), considerando el uso de la lengua como un fenómeno semióticosocial, explora el poder de la gramática para: crear significado (realizando el "significado potencial" de la lengua) y cambiar nuestras vidas.

De todo esto, deducimos que el discurso, con esta base de interacción cognitivamental, establece una dinámica argumentativa (de naturaleza retórica): construyendo, mediante el uso de la lengua, la perspectiva propia con respecto a la perspectiva del otro. Y podríamos plantear, mediante elementos matemáticos, la siguiente operación mental, que establece una dinámica argumentativa en el discurso:

DISCURSO $=[$ perspectiva propia \pm perspectiva del otro $]=$ Dinámica Argumentativa

Figura 4. Dinámica argumentativa en el discurso

Así, por ejemplo, el texto del mensaje dejado en el servicio del contestador telefónico [Hello Lázaro, it's Thèrese. I'll try you again later. Nothing important. Thank you!. Bye!] nos muestra como Thèrese asume dos perspectivas: la suya al construir su mensaje, y la del destinatario del mensaje, la cual se hace evidente por el uso deíctico del pronombre you, y 
sobre todo por la expresión Nothing important. Esta expresión actúa como conclusión de una dinámica argumentativa donde las premisas están implícitas, y sólo las maneja el emisor del mensaje.

Por consiguiente, podemos afirmar que discurso es una práctica compleja, no ya sólo por su proceso comunicativo, sino también por su carácter de intención cognitiva y social. Práctica que realizamos a diario, y que necesitamos realizar, y donde la lengua es cargada con una determinada fuerza comunicativa (sociocultural e ideológica) y estructurada en una mutua dependencia con esta fuerza. Así pues, discurso está constituido por una construcción semántico-pragmática, y por una concreción gramático-retórica (el texto), y en consecuencia provisto de:

a) una función comunicativa, donde interviene: la situación de comunicación,

b) una fuerza ilocutiva, donde interviene la intención del hablante y la percepción del oyente, y el aspecto cognitivo del: - conocimiento compartido, - conocimiento individual, y - conocimiento que uno tiene del otro.

\section{A MODO DE CONCLUSIÓN}

El discurso es quien desarrolla o explota la lengua, funcionando en una variedad de diferentes contextos socioculturales. Para los antropólogos el estudio de la acción de la lengua es el estudio del poder, de la ideología y del cambio cultural; y desde esta perspectiva se ha subrayado la naturaleza de la lengua como discurso. Para nosotros, desde esta perspectiva, la acción de la lengua (el discurso) tiene una orientación argumentativa, en una dinámica de perspectivas de diálogo y de interacción.

Fowler (1996: 93-94) afirma que la lengua en uso real es más que un texto, es un discurso, y ver la lengua como discurso es estudiarla en su contexto comunicativo social e históricamente situada. De este modo, Fowler aclara que la estructura del discurso en contraposición a la estructura del texto, más limitada, refleja el íntegro y complejo proceso de la gente interaccionando en situaciones llenas de vida y dentro de la estructura de las fuerzas sociales. Así, la lengua, como el discurso, expresa mediante su extra-estructura las interacciones personales en la comunicación, las relaciones y posiciones sociales de los hablantes, y la naturaleza del escenario en el que la lengua es usada. Nosotros consideramos que estos elementos -interacción personal, relación y posición social, y escenario de uso- intervienen en la naturaleza argumentativa del uso de la lengua.

Con todo ello, podemos afirmar que discurso es la acción comunicativa de la lengua, y texto la organización y estructuración de esa acción comunicativa. Todo lo asignado al estudio del texto, es asignado también al estudio del discurso. La lengua es discurso. Su análisis requiere, a la vez, un análisis lingüístico y un análisis de los aspectos del contexto situacional y cultural.

Además, es necesario resaltar el carácter de evento social que el discurso tiene. Y como tal, cada acto discursivo, hablado o escrito, siempre tiene un efecto. Un efecto que puede ser considerado como un acto de poder que caracteriza al uso de la lengua disponible en cada ocasión. Y estimamos que las categorías semánticas (o metafunciones de la lengua) conceptual, interpersonal, textual- y las categorías situacionales -field, tenor, mode- de Halliday, circunscriben todo tipo de discurso a su naturaleza retorico-argumentativa. 
Así, la Figura 2. Relación discurso-texto en las funciones de la lengua puede ser completada de la siguiente forma:

\begin{tabular}{|c|c|c|c|}
\hline Contexto de la & ación & PROCESO de: & Funciones de la lengua \\
\hline "Field" & 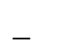 & construcción de la realidad & Conceptual \\
\hline "Tenor" & - & interacción social & Interpersonal \\
\hline "Mode" & - & $\begin{array}{c}\text { cómo es usada la lengua } \\
\text { PRODUCTO de: }\end{array}$ & Textual \\
\hline
\end{tabular}

Figura 5. Proceso (Discurso) y Producto (Texto) de la Lengua

Por tanto, el discurso es un proceso que caracteriza a la lengua por la intervención o interacción de sus usuarios en un determinado tiempo y lugar, con un propósito, y en un contexto verbal, situacional, y cultural.

\section{REFERENCIAS BIBLIOGRÁFICAS}

Adam, J-M. 1997 ( $3^{\mathrm{a}}$ ed.). Les textes: types et prototypes. Récit, description, argumentation, explication et dialogue. Paris: Nathan.

Bakhtin, M. 1986. Speech Genres and Other Late Essays. Trad. Vern W. McGee. Austin, TX.: University of Texas Press.

Beaugrande, R. 1980. Text, Discourse and Process. Norwood, NJ.: Ablex Publishing Corporation.

1984. Text Production. Norwood, NJ.: Ablex Publishing Corporation

Beaugrande, R. \& W. Dressler. 1981. Introduction to Text Linguistics. London, New York: Longman.

BIRCH, D. 1996. "Critical linguistics as cultural process". En J. JAMES, ed., The Language Culture Connection. Singapore: SEAMEO Regional Language Centre

Blakemore, D. 2002. Relevance and Linguistic Meaning. The Semantics and Pragmatics of Discourse Markers. Cambridge: CUP.

Brown, G. \& G. Yule. 1983. Discourse Analysis. Cambridge: CUP.

CARTER, R. 1997. Investigating English Discourse. Language, literacy and literature. London, New York: Routledge.

Chatman, S. 1978 (5 impression: 1989). Story and Discourse. Narrative Structure in Fiction and Film. Ithaca, London: Cornell University Press.

Cook, G. 1989. Discourse. Oxford: Oxford University Press.

Dik, S.C. 1997. The theory of Functional Grammar. [TFG] Ed. Kees Hengeveld. Part 1, The Structure of the Clause ( $2^{\text {nd }}$ ed. rev.) [TFG1]; Part 2, Complex and Derived Constructions. [TFG2]. Berlin : Mouton de Gruyter.

Edmonson, W. 1981. Spoken Discourse. A model for analysis. London, New York: Longman. 
EDwARDs, D. 1997. Discourse and Cognition. London: SAGE Publications.

EnKvist, N. E. 1991. “'Discourse strategies and discourse types”. En E. Ventola ed., Functional and Systemic Linguistics. Approaches and Uses. Berlin, New York: Mouton de Gruyter. 3-22.

Fairclough, N. 1995. Media Discourse. London, New York: Edward Arnold. 2003. Analysing Discourse: Textual Analysis for Social Research. London: Routledge.

FawCEtT, R. P. 1980. Cognitive Linguistics and Social Interaction. Towards an integrated model of a systemic functional grammar and the other components of a communicating mind. Heidelberg: Julius Groos Verlag and Exeter University.

Fowler, R. 1996 ( $2^{\text {nd }}$ edition / $1^{\text {a }}$ ed.: 1986). Linguistic Criticism. Oxford/New York: Oxford University Press.

Gernsbacher, M. A. y T. Givón, eds. 1995. Coherence in Spontaneous Text. Amsterdam, Philadelphia: John Benjamins.

Givón, T. 1984. Syntax. A Functional-Typological Introduction. Vol. I. Amsterdam, Philadelphia: John Benjamins.

Halliday, M. A.K. 1978. Language as a Social Semiotic. The Social Interpretation of Language and Meaning. London: Edward Arnold.

Arnold. 1994 (2 ed.; $1^{a}$ ed.: 1985). Functional Grammar. London: Edward

. 2002a. On Grammar. London: Jonathan J. Webster.

Webster.

Halliday, M. A.K. \& R. Hasan. 1976 (12 impression: 1993). Cohesion in English. London, New York: Longman.

1985 (reimpreso 1998). Language Context and Text: Aspects of Language in a Social-Semiotic Perspective. Greelong, Victoria: Deakin University Press.

Hodge, R. \& G. Kress. 1988 (reimpreso en 1995). Social Semiotics. Cambridge: Polity Press.

Hymes, D. H. 1986. "Models of the interaction of language and social life". Eds. J. J. Gumper y D. Hymes. Directions in Sociolinguistics: the Ethnography of Communication. Oxford: Basil Blackwell. 35-71.

JAwORSki, A. \& N. Coupland, N. 1999. "Introduction: Perspectives on discourse analysis". En A. Jaworski, y N. Coupland eds., The Discourse Reader. London, New York: Routledge. 1-53.

KinneAvy, J. L. 1971 (1980). A Theory of Discourse. New York, London: Norton \& Company. 
Kress, G., R. Leite-García \& T. Van Leeunen. 1997. "Discourse Semiotics". Ed. T. A. van Disk. Discourse as Structure and Process. Discourse Studies: A Multidisciplinary Introduction Volume 1. London: SAGE Publications. 257-291.

Kress, G. y Leeuwen, T. Van. 2001. Multimodal Discourses. London: Arnold.

LemKe, J. L. 1991. “Text production and dynamic text semantics”. Ed. E. Ventola. Functional and Systemic Linguistics. Approaches and Uses. Berlin, New York: Mouton de Gruyter. 23-38.

Leech, G. N. y Short, M. H. 1981. Style in Fiction. London, New York: Longman.

Martínez-CABeZA, M. A. 2002. The study of Language beyond the sentence: From Text Grammar to Discourse Analysis. Granada: Comares.

Martínez-DueÑas, J. L. 2002. Retórica de la lengua inglesa. Granada: Comares.

Melrose, R. 1988. "Systemic linguistics and the communicative language syllabus". Eds. R. Fawcett y D. Young. New Developments in Systemic Linguistics. Volume 2: Theory and application. London, New York: Pinter Publishers. 78-93.

Mills, S. 1997. Discourse. London: Rouletdge.

Nystrand, M. 1986. The Structure of Written Communication. Studies in Reciprocity between Writers and Readers. London: Academic Press.

Quirk, R., S. Greenbaum, G. Leech. \& J. Svartvik. 1985. A Comprehensive Grammar of English. London, New York: Longman.

Sanders, T. J. M. \& M.A. Gernsbacher. 2004. "Accessibility in Text and Discourse Processing”. Discourse Processes, 37(2). 79-89.

SCHIFFrIn, D. 2002 (1994). Approaches to Discourse. Oxford/Cambridge, Mass. Basil Blackwell.

SinClair, J. M. 1992. "Priorities in discourse analysis". Ed. M. Coulthard. Advances in Spoken Discourse Analysis. London: Routledge. 79-88.

Steiner, E. H. \& R. Veltman. 1988. "Introduction”. Eds. E. H. Steiner \& R. Veltman. Pragmatics, Discourse and Text. Some Systemically-inspired Approaches. London: Pinter Publishers. 1-12.

Stubbs, M. 1983. Discourse Analysis: The Sociolinguistic Analysis of Natural Language. Oxford: Basil Blackwell.

Virtanen, T., ed. 2004. Approaches to Cognition through Text and Discourse. Berlin, New York: Mouton de Gruyter.

Werlich, E. 1982 (1 $1^{\text {a }}$ ed. 1976). A Text Grammar of English. Heidelberg: Quelle \& Meyer.

Werth, P. 1999. Text Worlds: Representing Conceptual Space in Discourse. New York: Longman.

Widdowson, H. G. 2004. Text, Context, Pretext. Critical Issues in Discourse Analysis. Vienna: University of Vienna. 